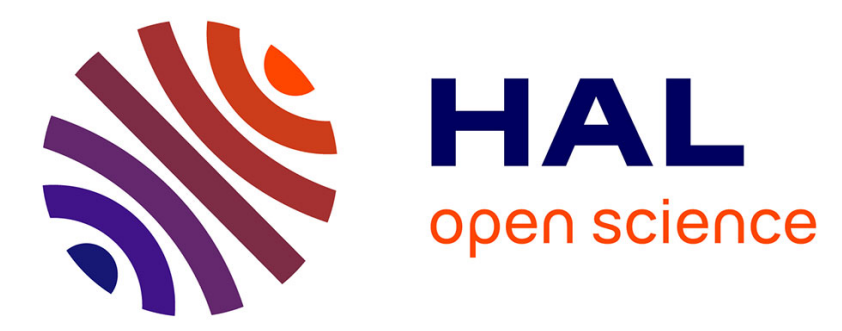

\title{
Water adsorption by a sensitive calibrated gold plasmonic nanosensor
}

Benjamin Demirdjian, Frédéric Bedu, Alain Ranguis, Igor Ozerov, Claude Henry

\section{- To cite this version:}

Benjamin Demirdjian, Frédéric Bedu, Alain Ranguis, Igor Ozerov, Claude Henry. Water adsorption by a sensitive calibrated gold plasmonic nanosensor. Langmuir, 2018, 34, pp.5381-5385. 10.1021/acs.langmuir.8b00040 . hal-01773221

\section{HAL Id: hal-01773221 https: / hal-amu.archives-ouvertes.fr/hal-01773221}

Submitted on 27 Apr 2018

HAL is a multi-disciplinary open access archive for the deposit and dissemination of scientific research documents, whether they are published or not. The documents may come from teaching and research institutions in France or abroad, or from public or private research centers.
L'archive ouverte pluridisciplinaire HAL, est destinée au dépôt et à la diffusion de documents scientifiques de niveau recherche, publiés ou non, émanant des établissements d'enseignement et de recherche français ou étrangers, des laboratoires publics ou privés. 


\section{Water adsorption by a sensitive calibrated gold plasmonic nanosensor}

Benjamin Demirdjian*, Frédéric Bedu, Alain Ranguis, Igor Ozerov, and Claude R. Henry

Aix-Marseille Univ, CNRS, CINaM, Marseille, France

To be published in Langmuir: DOI: 10.1021/acs.langmuir.8b00040

https://pubs.acs.org/doi/10.1021/acs.langmuir.8b00040

*Corresponding author:

B. Demirdjian, Aix-Marseille Univ., CNRS, CINaM UMR 7325, 13288 Marseille, France mobile:

+33 (0)6 6036 2818, fax: +33 (0)4 9141 8916, e-mail: demirdjian@cinam.univ-mrs.fr 


\begin{abstract}
We demonstrate in this work that using nanoplasmonic sensing it is possible to follow the adsorption/desorption of water molecules on gold nanodisks nanofabricated by electron beam lithography. This quantitative method is highly sensitive allowing the detection of a few hundredths of adsorbed monolayer. Disk parameters (height, diameter, inter-disk distance) have been optimized after finite-difference time-domain (FDTD) simulations in order to obtain the best localized surface plasmon resonance (LSPR) signal-to-noise ratio. Finally, we have precisely measured the adsorption kinetics of water on gold as a function of the relative humidity of the surrounding medium.
\end{abstract}

KEYWORDS: Nanoplasmonic, optical properties, water adsorption, UV-VIS spectroscopy, gold nanodisks, LSPR, EBL, FDTD simulations. 


\section{INTRODUCTION}

Progress in nanotechnologies needs to cross different scientific fields, in particular surface chemistry, nanophotonics and nanofabrication are largely employed in the development of chemical $^{1,2}$ and biological ${ }^{3-6}$ plasmonic resonance nanosensors. Plasmons, collective oscillations of electrons in noble metals, have resonant frequencies corresponding to visible and near infrared spectral regions for silver and gold. When gas or liquid molecules are adsorbed on the surface of noble metal thin films or nanoparticles, the minute local changes in refractive index strongly modify the optical response corresponding to surface plasmon resonance (SPR) in metal films and localized surface plasmon resonance (LSPR) in metal nanoparticles. Consequently, the spectral positions of the SPR or LSPR extinction peak, corresponding to the reflection minima or to the absorption maxima, are shifted following a simple $\operatorname{model}^{7}$ :

$$
\Delta \lambda=m\left(n_{2}-n_{1}\right)\left[1-\exp \left(\frac{-2 d}{l_{d}}\right)\right] .
$$

where $\Delta \lambda$ is the shift in wavelength in the extinction spectra corresponding to the plasmonic resonance, when the refractive index of surrounding media changes from $n_{1}$ to $n_{2}, m$ is the refractive index sensitivity. The parameters under the exponent $d$ and $l_{d}$ correspond, respectively, to the effective thickness of the adsorbed layer (medium with RI $n_{2}$ ) and to the evanescent electromagnetic field decay characteristic length. This characteristic decay length $l_{d}$ depends on the properties of the sensor material as well as on its geometrical size and shape.

Both types of sensors, based on SPR or LSPR are largely used for the detection and monitoring of RI changes. For thin film surface plasmon resonance sensors the typical values for the sensitivity $m$ are about $2 \times 10^{6} \mathrm{~nm}$ per refractive index unit (RIU) ${ }^{7}$ and $l_{d}$ are about 200-300 $\mathrm{nm}^{8}$ The reported refractive index sensitivities of typical LSPR sensors are more than $10^{4}$ times lower $\left(m \sim 2 \times 10^{2}\right.$ $\mathrm{nm} / \mathrm{RIU})$. However, this drawback is partially compensated by a shorter characteristic field decay length $l_{d}$ of about $5-40 \mathrm{~nm}^{2,8-12}$

Water interaction with metal surface has become one of the most widely studied fields in surface science $^{13}$. Motivations for these studies are almost as numerous as the number of studies 
themselves. For example, it is crucial for environmental chemistry, catalysis, biology, material corrosion, and electro-chemistry in which $\mathrm{H}_{2} \mathrm{O}$ is the most-used solvent. Despite the numerous studies on the interaction of water with gold surfaces ${ }^{14-17}$ controversy still remains concerning the adsorbed water layer thickness. In these studies several techniques (temperature-programmed desorption, X-ray photoelectron spectroscopy, atomic force microscopy, scanning Kelvin probe force microscopy, thermal desorption mass spectrometry) were used on single crystal and polycrystalline gold surfaces. These authors ${ }^{14-17}$ have measured water layer thicknesses going from a single bilayer to several nanometers. Here we present a method to measure with high accuracy the thickness of the water layer adsorbed on the surface of gold nanodisks.

\section{EXPERIMENTAL SECTION}

Gold nanodisk arrays were fabricated by electron-beam lithography (EBL) followed by a lift-off process. Borosilicate glass round substrates with a diameter of 1 inch $(25 \mathrm{~mm})$ and a thickness of 1 mm (from UQG Optics) were cleaned by successive immersion in acetone and isopropanol ultrasonic baths for 5 minutes in each solvent. Then the substrates were rinsed in deionized water and dried under clean nitrogen flow followed by an exposure to oxygen plasma at a temperature of $150^{\circ} \mathrm{C}$ in order to remove the remaining solvent traces and moisture, to activate the glass surface and to enhance the adhesion of the e-beam resist to the substrates. We used an electron beam resist (PMMA diluted in ethyl-lactate at 2\%, from Allresist) spin-coated at $4000 \mathrm{rpm}$ in order to obtain the final thickness of about $70-80 \mathrm{~nm}$. Then the resist was baked for 10 minutes at $170^{\circ} \mathrm{C}$ on a hotplate. In order to prevent charging of the dielectric substrates during EBL, the second layer, a conductive polymer (SX AR PC 5000/90.2 from Allresist, Germany) was spin-coated on the samples (rotation speed of $4000 \mathrm{rpm}$ ) and baked for 2 minutes at $90^{\circ} \mathrm{C}$ on a hotplate. The nanodisk arrays were patterned using Pioneer EBL tool (Pioneer, Raith, Germany) at following operation conditions: acceleration voltage of electron gun was $20 \mathrm{kV}$, the typical beam currents were about 27 pA for the aperture of $15 \mu \mathrm{m}$. The typical working distance was about $7 \mathrm{~mm}$, and the nominal 
exposition dose was chosen to be $90 \mu \mathrm{C} / \mathrm{cm}^{2}$ in order to prevent possible proximity effects. After the e-beam exposure, the conducting layer was washed out in pure water and the features were developed for 55s in 1:3 MIBK:isopropanol mixture followed by stopping in pure isopropanol and rinsing in pure water to remove the solvent traces. The thickness of the PMMA layer was measured to be $80 \mathrm{~nm}$ by a contact profilometer (DektakXT, Bruker, Germany) after e-beam exposure and resist development. Two successive metal layers (chromium for seeding and gold) were evaporated under vacuum conditions (Auto 306, Edwards, UK). The thickness of deposited bi-layers was monitored in situ by a quartz crystal microbalance and controlled by a contact profilometer once the lift-off process was finished. The lift-off process was started in acetone and then finished in pure ethyl lactate bathes, followed by rinsing and drying of the samples under nitrogen flow.

The EBL technique permits to control precisely the shape, the size and the distance between the gold nanodisks. We patterned typical square surfaces of $1 \mathrm{~mm}$ x $1 \mathrm{~mm}$ using a write field of $100 \mu \mathrm{m}$ x $100 \mu \mathrm{m}$ repeated with a stitching precision better than $20 \mathrm{~nm}$. Atomic force microscopy measurements (see figure 3) showed that the gold nanodisks have a center-to-center distance pitch $\langle p\rangle=300 \mathrm{~nm}$, an average height $\langle h\rangle=29 \mathrm{~nm}$, and an in-plane diameter $d=150 \mathrm{~nm}$ (the in-plane lengths are less accurate than the height measured by AFM).

The experimental device used for LSPR measurements has been described into details in reference $^{18}$.

\section{RESULTS AND DISCUSSION}

Before the fabrication of gold nanodisks by electron beam lithography (EBL) we have determined the disks characteristics (diameter, thickness, inter-disk distance) giving the best theoretical LSPR responses. For that purpose we perform finite-difference time-domain (FDTD) calculations (software OptiFDTD from Optiwave: https://optiwave.com/resources/academia/free-fdtddownload/) in order to have the parameters $p, d, h$ (figure 1) giving the best theoretical LSPR 
signal-to-noise ratio. We perform FDTD calculations with box dimensions of $0.50 \mu \mathrm{m}$ (length) * $0.30 \mu \mathrm{m}$ (width) * $0.30 \mu \mathrm{m}$ (depth). For $\mathrm{X}$ and $\mathrm{Y}$ directions we have chosen Periodic Boundary Conditions (PBC), for the $\mathrm{Z}$ direction we have chosen a Perfectly Matched Layer (PML) boundary condition.

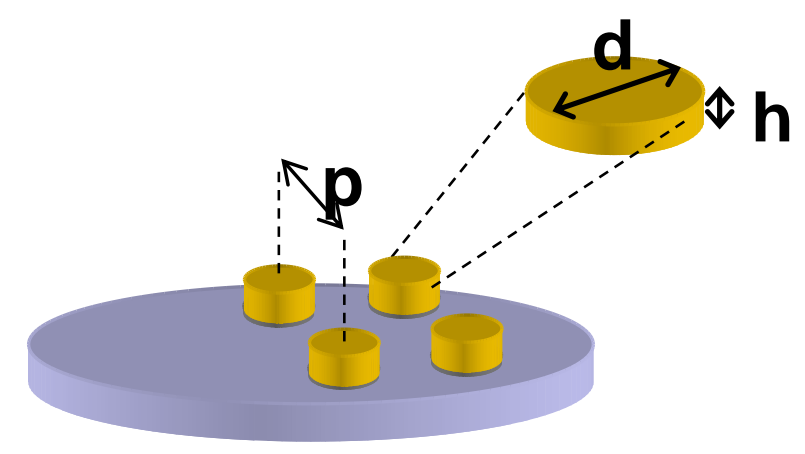

Figure 1: gold nanodisks deposited on a borosilicate glass window with parameters: pitch $p$ (center-to-center distance), diameter $d$, height $h$

Since there are 3 parameters $(p, d, h)$ we have performed 3 series of FDTD calculations keeping each time two of the three parameters constant and modifying only the third parameter (figures $2 \mathrm{a}$, $2 \mathrm{~b}, 2 \mathrm{c}$ ). These calculations show that the diameter $d$ of the nanodisks is a crucial parameter (figure 2c, table 1). Indeed, when $d$ increases, the position of the peak is strongly red-shifted and its intensity and FWHM increase significantly (figure $2 \mathrm{c}$ ). For a diameter $d=50 \mathrm{~nm}$, the $\mathrm{S} / \mathrm{N}$ ratio is too low to correctly determine the FWHM of the LSPR peak (figure 2c). Out of possible diffractive coupling between the disks, the parameter $p$ has little effect on the position of the LSPR peak ${ }^{19}$. However, increasing the parameter $p$ leads to a narrowing of the peak (table S1) as it has been seen by Jiang et al. ${ }^{20}$. Finally, the height $h$ of the gold nanodisks substantially modifies the peak position and intensity but has little effect on its width (figure $2 b$, table S2). These results are consistent with the works of Zheng et al. ${ }^{21}$ and Chen et al. ${ }^{10}$ showing that the fundamental parameters are $d$ and $h$, and more precisely the aspect ratio $d / h$ defined as the diameter-to-height ratio. These authors have shown that gold nanodisks with high aspect ratio lead to a larger signal-to-noise ratio and a better sensibility of the refractive index ( $m$ from equation (1)). They concluded also that increasing the Au 
disk diameter results in a redshift of the peak and increases both its intensity and width. Finally, reducing the thickness of the Au nanodisks leads to a redshift of the LSPR peak which becomes narrower and higher. These conclusions are coherent with our results.

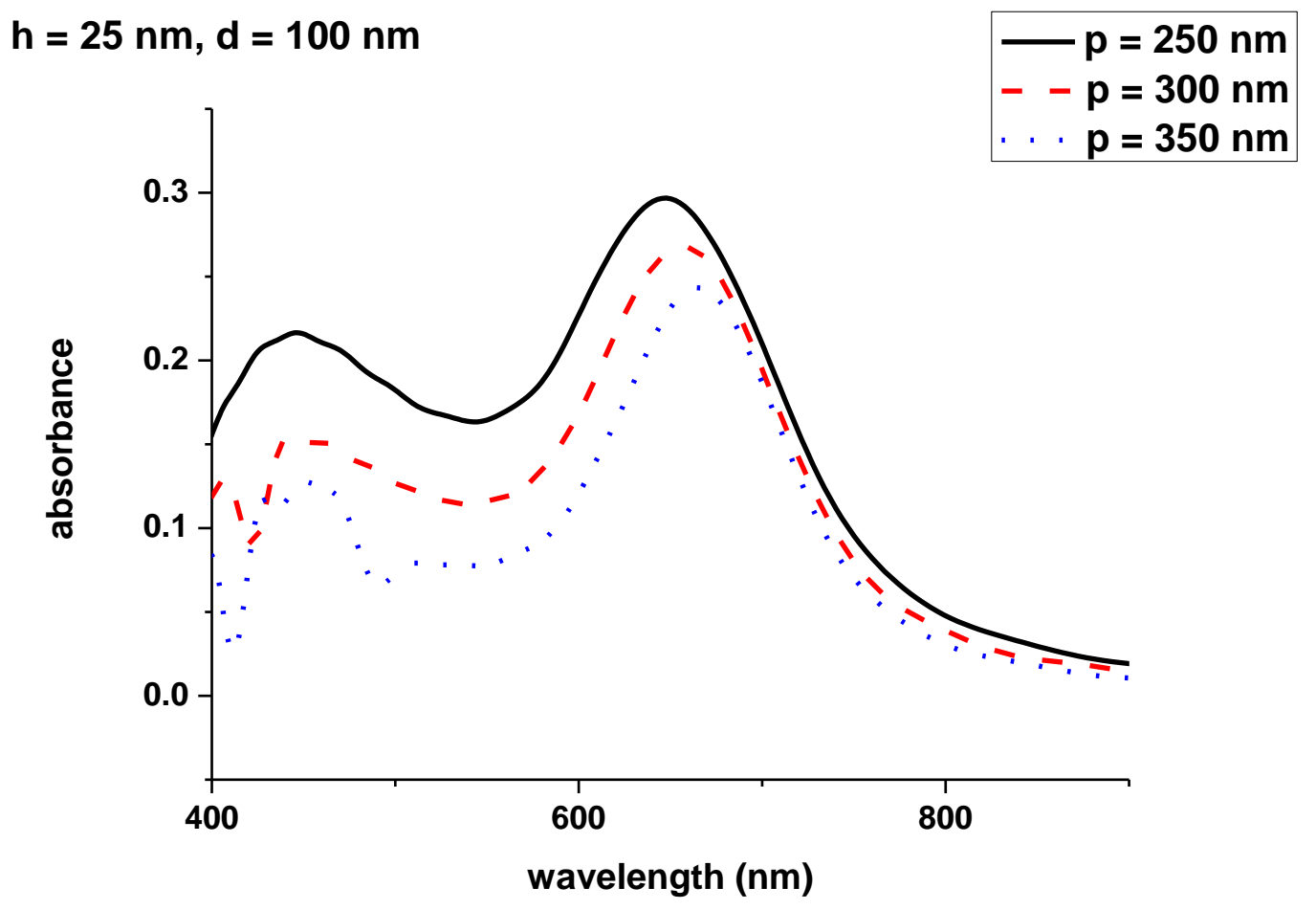

Figure 2a: Simulated LSPR responses for gold nanodisks ( $h=25 \mathrm{~nm}, d=100 \mathrm{~nm})$ deposited onto a borosilicate glass window vs the pitch $p$.

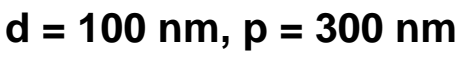
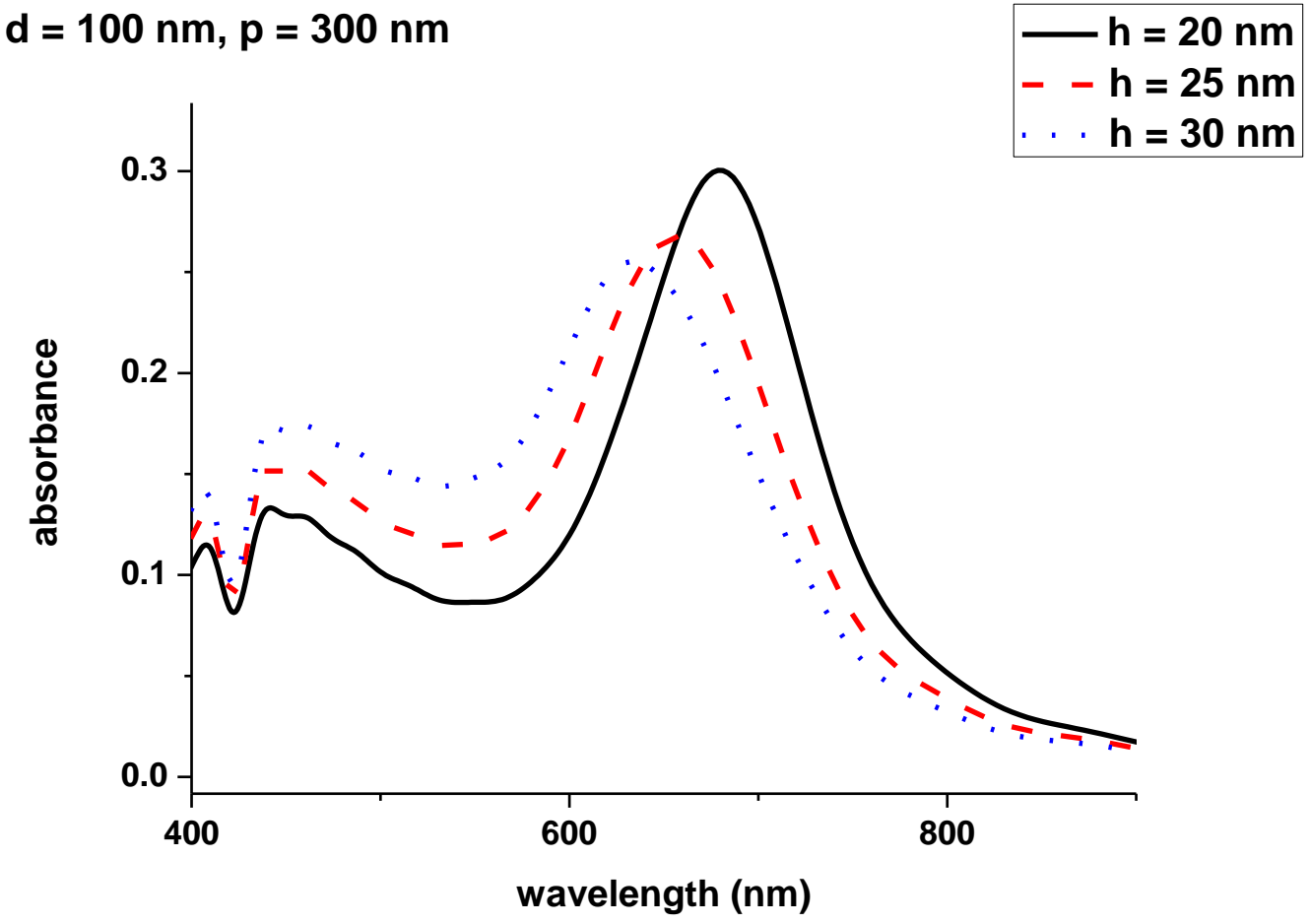

Figure 2b: Simulated LSPR responses for gold nanodisks ( $d=100 \mathrm{~nm}, \mathrm{p}=300 \mathrm{~nm})$ deposited onto a borosilicate glass window vs the height $h$. 


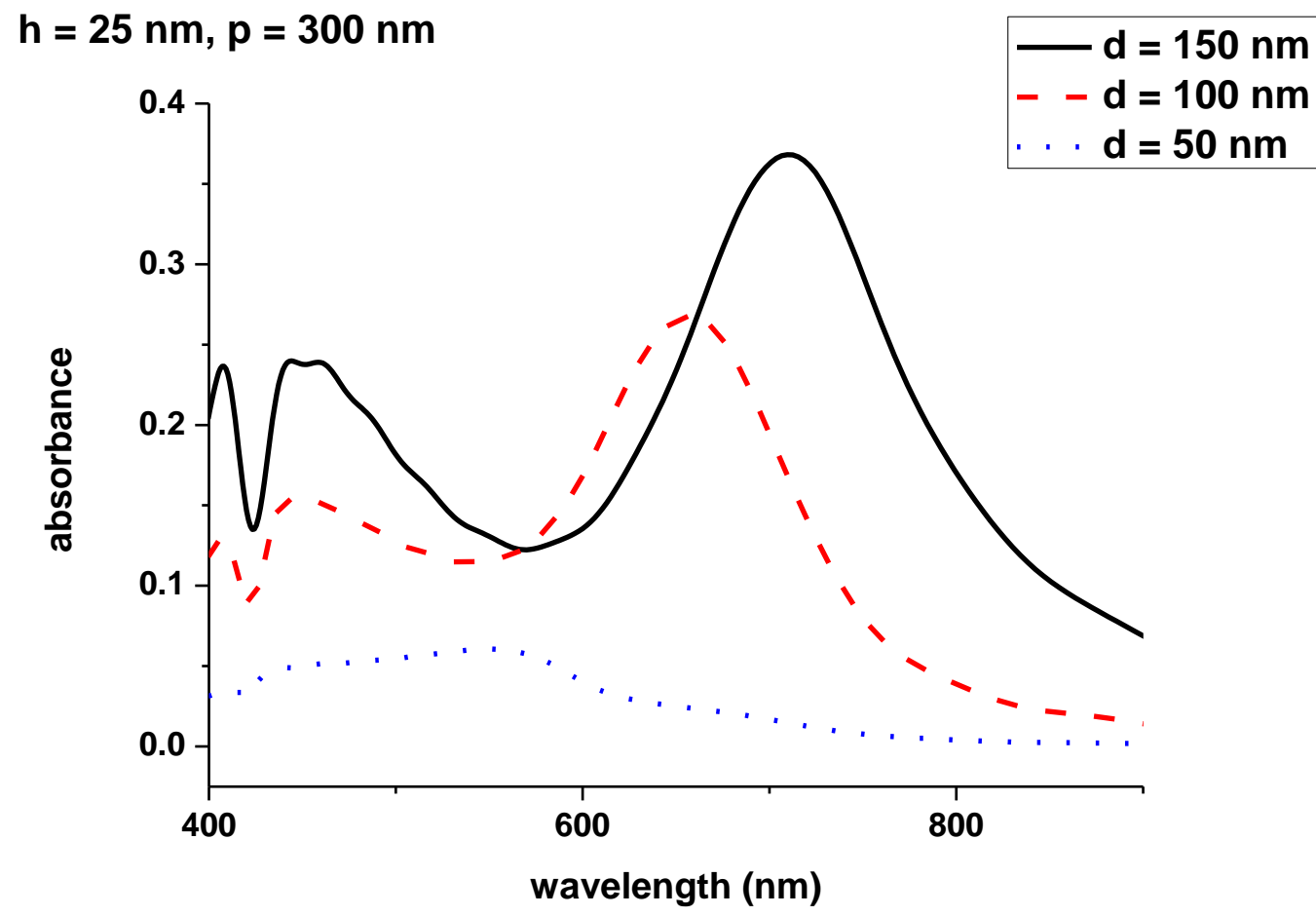

Figure 2c: Simulated LSPR responses for gold nanodisks $(h=25 \mathrm{~nm}, p=300 \mathrm{~nm})$ deposited onto a borosilicate glass window vs the diameter $d$.

Table 1: Simulated LSPR peak positions ( $\lambda$ ) and FWHM of the curves of the figure $2 c$

\begin{tabular}{|c|c|c|c|c|c|}
\hline$d$ & $p$ & $h$ & $d / h$ & $\lambda$ & $F W H M$ \\
\hline 50 & 300 & 25 & 2 & 548 & n.d. \\
\hline 100 & 300 & 25 & 4 & 656 & 95 \\
\hline 150 & 300 & 25 & 6 & 710 & 114 \\
\hline
\end{tabular}

These last results confirm that the best LSPR experimental responses should be obtained with a high aspect ratio, i.e. for wide and thin gold nanodisks. Using the PLANETE cleanroom facility we have fabricated two different types of samples (figure 3). We show clearly that $d$ is really a critical parameter on the experimental LSPR responses (figure 3) confirming the theoretical results obtained by FDTD calculations (figure 2c, table 1). 


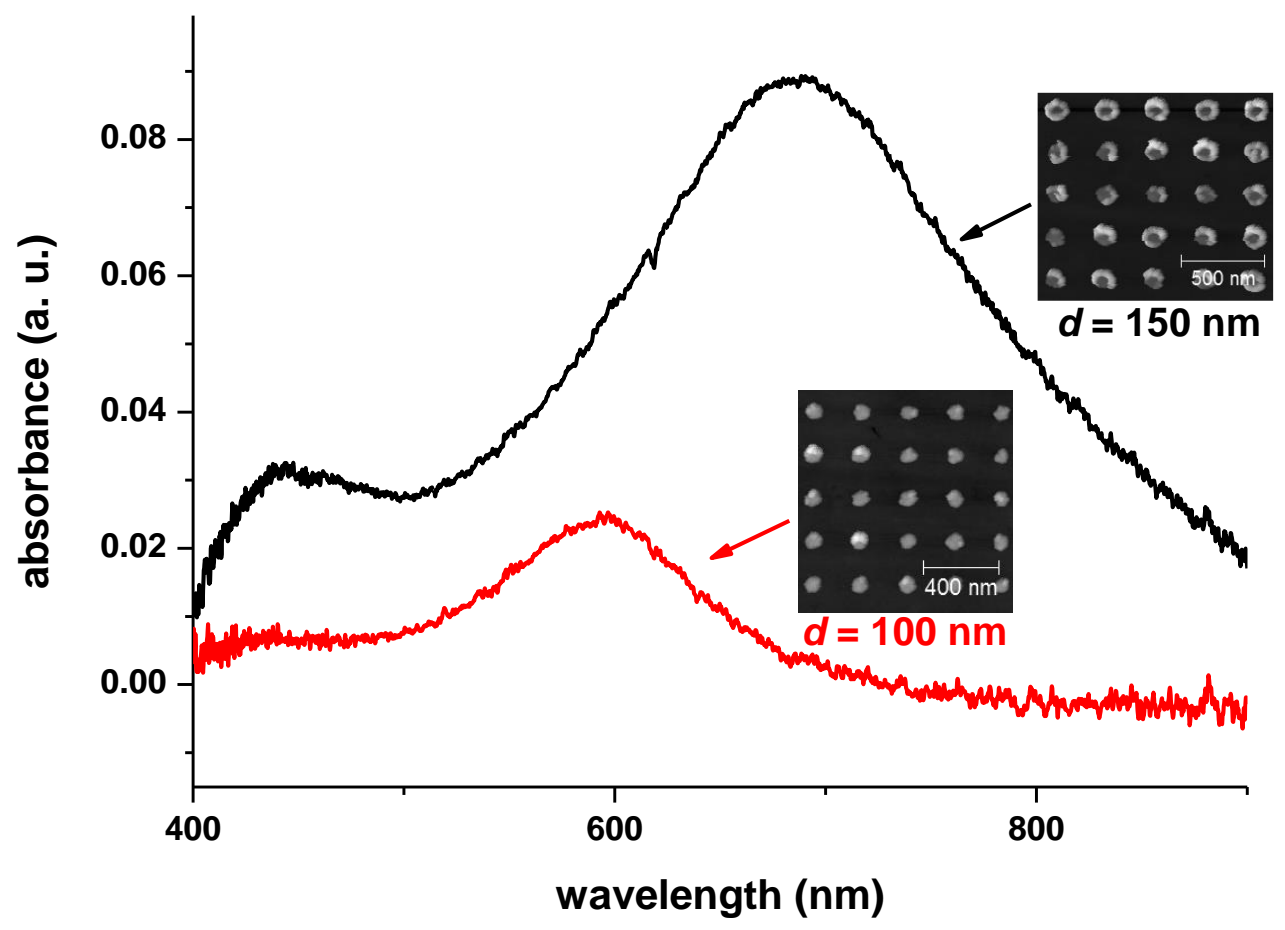

Figure 3: Experimental LSPR responses for gold nanodisks $(\langle h\rangle=29 \mathrm{~nm},\langle p\rangle=300 \mathrm{~nm}$ ) deposited onto a borosilicate glass window vs the diameter $d$. Tapping mode topographical AFM image of gold nanodisks with $d=100$ and $150 \mathrm{~nm}$. Images obtained with a PSIA apparatus XE-100.

For gold disks with $d=150 \mathrm{~nm}$, the position of the LSPR experimental peak $(\lambda=687 \mathrm{~nm})$ is blueshifted compared to the theoretical peak position of the figure $2 \mathrm{c}(\lambda=710 \mathrm{~nm})$. This is partially due to the fact that the actual height of our gold nanodisks, measured by AFM, is $\langle h\rangle=29 \mathrm{~nm}$ whereas a height $h=25 \mathrm{~nm}$ was considered in the FDTD simulations. Indeed, we have seen in figure $2 \mathrm{~b}$ that thicker nanodisks lead to a blue-shifted LSPR signals. FDTD simulations performed with a parameter $h=29 \mathrm{~nm}$ (see figure S1) give also a LSPR peak position at $\lambda=692 \mathrm{~nm}$ closer to the experimental value $(\lambda=687 \mathrm{~nm})$.

\section{Calibration of $m$ and $l_{d}$}

Using equation (1), it is possible to precisely measure $m$, the sensibility of the refractive index, by varying the refractive index $n$ of the surrounding medium of our sample. We took 4 surrounding media with 4 different indices $n: n=1$ (air), $n=1.33$ (water), $n=1.36$ (ethanol), $n=1.43$ (ethylene glycol). When the sample is immersed into the different liquids, equation (1) is simplified to $\Delta \lambda \sim m$ 
$\Delta n$ because the thickness of the adsorbate layer $d$ is considered to be infinite. Thus, we obtain a linear relation between $\Delta \lambda$ and $\Delta n$ which $m$ is the slope (figure 4). We find a value of $m=163 \mathrm{~nm} \pm$ $3 \mathrm{~nm} / \mathrm{RIU}$ which is in good agreement with references ${ }^{2,8-12}$ and close to the theoretical value obtained by FDTD simulations on this system ( $m=184 \mathrm{~nm} \pm 14 \mathrm{~nm} / \mathrm{RIU}$, see figure S2).

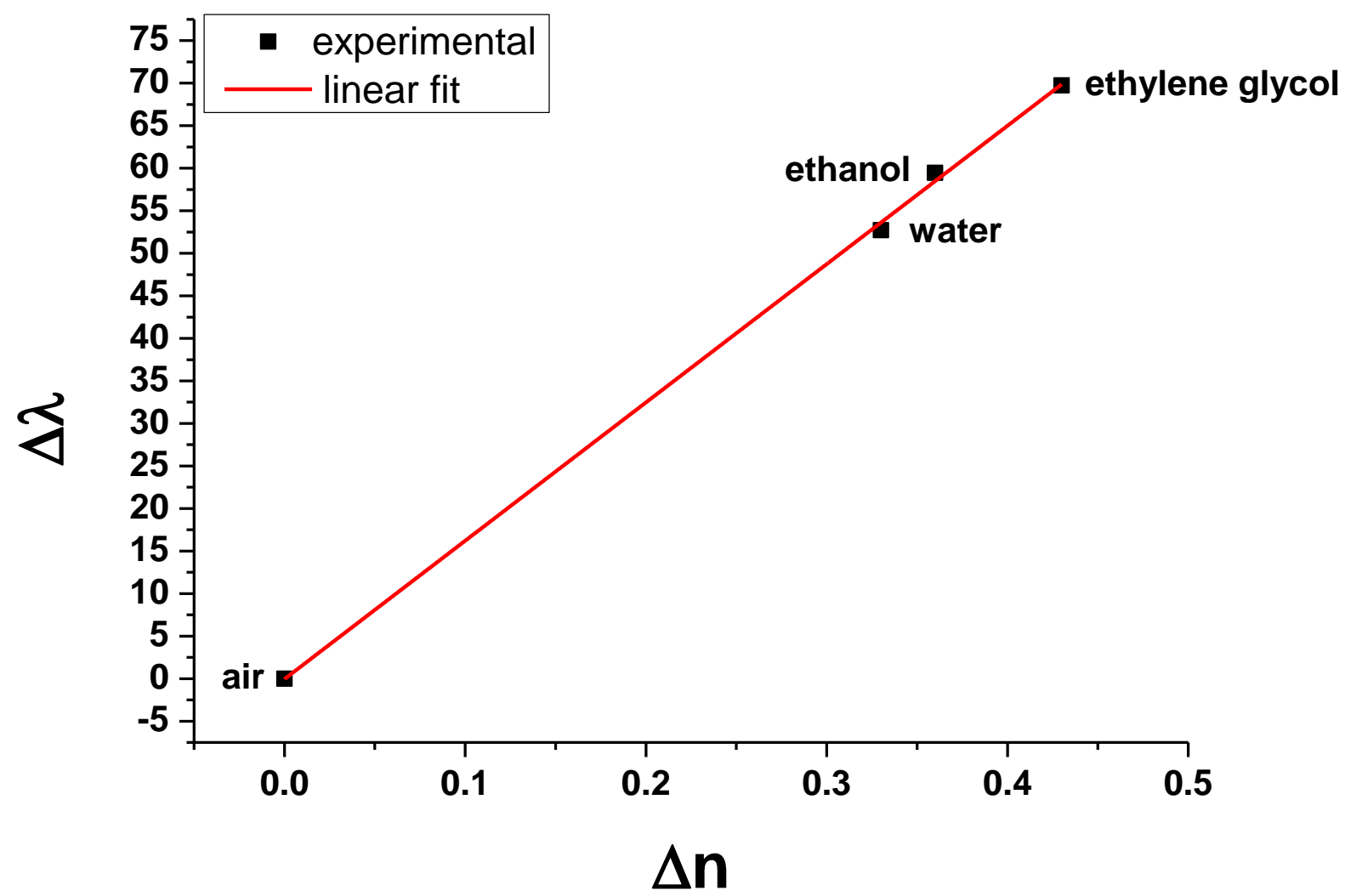

Figure 4: Evolution of the shift $\triangle \lambda$ of the LSPR response of gold nanodisks deposited on a borosilicate glass window vs the refractive index of the surrounding medium (air, water, ethanol, ethylene glycol).

In order to determine $l_{d}$, the characteristic decay length of the evanescent electromagnetic field, we deposited a $8 \mathrm{~nm}$ thick layer of $\mathrm{SiO}_{\mathrm{x}}$ of by radio-frequency (RF) magnetron sputtering of pure $\mathrm{SiO}_{2}$ target using a system (ALCATEL SMC600, France), operating at RF power of 400W under argon gas atmosphere (purity $\geq 99.999 \%$ ), the gas flux was of $10 \mathrm{sccm}$ and the working pressure was 0.8 $\mathrm{Pa}\left(8.10^{-3}\right.$ mbar $)$.

It induces a shift $\Delta \lambda=27 \mathrm{~nm}$. From equation (1), taking $n_{1}=1$ (vacuum), $n_{2}=1.47\left(\mathrm{SiO}_{2}\right)$ and $m$ $=163 \mathrm{~nm} / \mathrm{RIU}$, one get a value of $l_{d}=37 \mathrm{~nm}$ in good agreement with literature ${ }^{2,8-12}$ for gold disks 
having the same aspect ratio values. Our system being well calibrated, it becomes possible to perform quantitative adsorption/desorption measurements and to deduce the thickness $(d)$ of the adsorbed film from the LSPR shift $\Delta \lambda$ from the equation (1). We have performed the adsorption/desorption measurements on the bare gold nanodisks, i.e. not covered by the $\mathrm{SiO}_{2}$ layer. Indeed the $\mathrm{SiO}_{2}$ layer decreases the sensitivity of the refractive index $m$ of $22 \%$, going down from $163 \mathrm{~nm} / \mathrm{RIU}$ to $127 \mathrm{~nm} / \mathrm{RIU}$.

\section{Water adsorption/desorption on gold nanodisks}

Before the introduction of the water vapor, the UHV reactor was pumped until a pressure $\mathrm{P}<10^{-6}$ mbar. The relative humidity $(R H$ in \%) in the UHV reactor is defined as the ratio of the water vapor partial pressure to the saturated water vapor pressure at a given temperature. Pure water from Fluka Chemie AG is further purified by the technique described in Ferry et al. ${ }^{22}$, water vapor is introduced into the chamber through a leak valve.

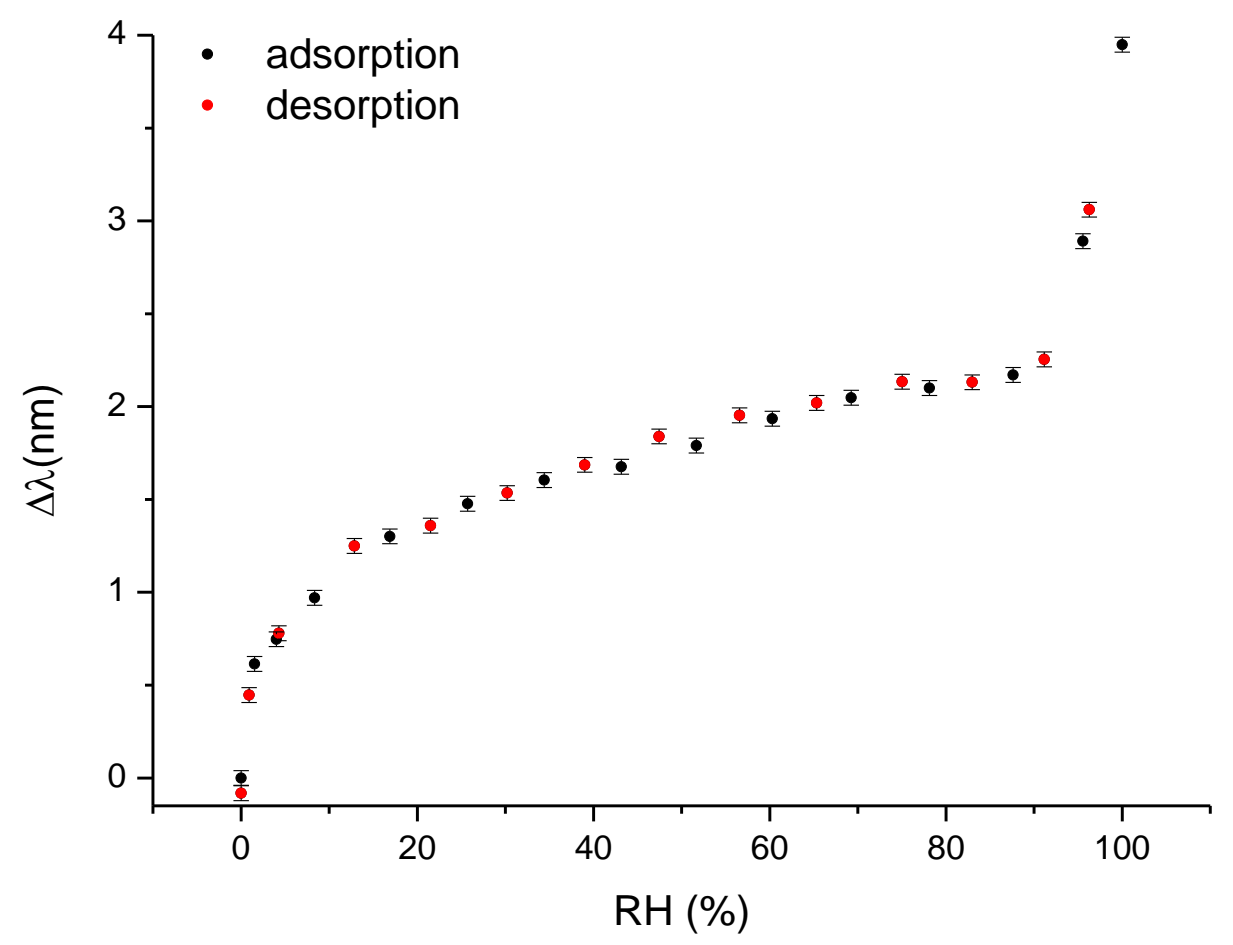

Figure 5: Evolution at room temperature of the shift $\Delta \lambda$ of the LSPR response vs the relative humidity inside the reactor during the adsorption of water vapor on the bare gold nanodisks. Black dots are obtained during the adsorption, red dots are obtained during the desorption. The accuracy of the wavelength measurements in the LSPR response is $0.04 \mathrm{~nm}$. 
Figure 5 shows the evolution of the shift $\Delta \lambda$ of the LSPR response $v s R H$ inside the reactor at room temperature. Between $20<R H<90 \%$ the shift $\Delta \lambda$ is almost linear $v s$ the relative humidity. At $R H$ $=100 \%$ we measure $\Delta \lambda_{\max }=3.95 \mathrm{~nm}$. From equation (1) this shift corresponds to a thickness of the adsorbed water layer of $d=1.41 \mathrm{~nm}$ i.e. 4.7 monolayers (ML) of liquid water assuming $0.3 \mathrm{~nm}$ per ML (mean van der Waals diameter of water). We observe also that at $R H \sim 10 \%, \Delta \lambda=1 \mathrm{~nm}$ corresponding to about 1 water monolayer. Guo et al. ${ }^{17}$ have measured by SKPFM the thickness of water films adsorbed on metal surfaces at different $R H$ from the force-distance curves. The thicknesses of the water film, for a given $\mathrm{RH}$, are different for copper, chromium and gold surfaces due to the differences in roughness and contact angle. For gold surfaces, they show a thickness of about $1 \mathrm{~nm}$ at $R H=100 \%$, i.e. about $3.3 \mathrm{H}_{2} \mathrm{O}$ ML. This is slightly smaller than the value we measure in figure 5 (4.7 ML), it could be explained by a by a different roughness of the gold deposits.

For this calculation we take the calibrated sensitivity of the refractive index $m=163 \mathrm{~nm} / \mathrm{RIU}$ and the calibrated characteristic decay length of the evanescent electromagnetic field $l_{d}=37 \mathrm{~nm}$. We assume that water condenses onto the gold surface as liquid water, so we take $n_{2}=n\left(\mathrm{H}_{2} \mathrm{O}_{\text {liq }}\right)=1.33$.

Gil et al. ${ }^{15}$ show that on polycrystalline gold surfaces water accumulates at low RH at the grain boundaries as puddles. At higher RH a uniform water film of several nm adsorbs on the terraces. We have probably on our sample this kind of adsorption phenomenon, ie. puddles or islands growing progressively on the gold surface before to have a completed water layer.

This technique is highly sensitive to the adsorption of water molecules on gold nanodisks, we are able to detect about $4 / 100$ of water monolayer !

Adsorption and desorption curves are reversible (figure 5), thus we expect physisorption and no chemisorption of water molecules onto the gold nanodisks. This is in good agreement with the work 
of Heras et al. ${ }^{16}$ showing no evidence of $\mathrm{H}_{2} \mathrm{O}$ dissociation on gold films between 77 and $373 \mathrm{~K}$ and the report of Henderson et al. ${ }^{13}$ where no dissociation was detected on gold single crystals.

Compared to our previous work (B. Demirdjian et al. ${ }^{18}$ ), we have now calibrated our plasmonic sensor therefore we can quantitatively measure the amount of water monolayers adsorbed onto gold nanodisks (figure 5). We have built a very sensitive calibrated humidity sensor able to detect a few hundredths of monolayer.

This plasmonic probe will be useful to follow the reactivity of metallic nanoparticles (Pt, $\mathrm{Pd}$, nanoalloys, ...) supported on thin oxide film deposited on gold disks with different gases involved in catalysis such as $\mathrm{CO}, \mathrm{O}_{2}, \mathrm{H}_{2}, \mathrm{NO}_{\mathrm{x}}$. Metallic nanoparticles can react with gas molecules that change the dielectric properties at its surface and induce a shift of the LSPR wavelength of the underlying gold nanodisk. This is called an indirect LSPR phenomenon or indirect nanoplasmonic sensing (INPS). This technique will allow probing quantitatively and with a high sensitivity the catalytic and photocatalytic activities of nanoparticles under various pressures and temperatures by coupling LSPR responses with mass spectrometry signals.

\section{CONCLUSIONS}

This work shows that the nanoplasmonic sensing lets us to follow the adsorption/desorption of water molecules on gold nanodisks fabricated by electron beam lithography. This new method is highly sensitive and quantitative and lets us to determine precisely the amount of water adsorbed onto the sensor surface. This is a promising method to follow the catalytic reactions on metallic nanoparticles deposited on the gold nanodisks by indirect nanoplasmonic sensing.

\section{ACKNOWLEDGMENTS}

Nanofabrication processes were performed in PLANETE cleanroom facility (CINaM, Marseille). 


\section{REFERENCES}

(1) Kalyuzhny, G.; Schneeweiss, M. A.; Shanzer, A.; Vaskevich, A.; Rubinstein, I. Differential plasmon spectroscopy as a tool for monitoring molecular binding to ultrathin gold films. $J$. Am. Chem. Soc. 2001, 123, 3177-3178.

(2) Malinsky, M.; Kelly, K.; Schatz, G.; Van Duyne, R. P. Chain length dependence and sensing capabilities of the localized surface plasmon resonance of silver nanoparticles chemically modified with alkanethiol self-assembled monolayers. J. Am. Chem. Soc. 2001, 123, 1471-1482.

(3) Connolly, S.; Cobbe, S.; Fitzmaurice, D. Effects of Ligand-Receptor Geometry and Stoichiometry on Protein-Induced Aggregation of Biotin-Modified Colloidal Gold. J. Phys. Chem. B 2001, 105, 2222-2226.

(4) Haynes, C. L.; Van Duyne, R. P. Nanosphere lithography: a versatile nanofabrication tool for studies of size-dependent nanoparticle optics. J. Phys. Chem. B 2001, 105, 5599-5611.

(5) Danilov, A.; Tselikov, G.; Wu, F.; Kravets, V.K.; Ozerov, I.; Bedu, F.; Grigorenko, A.N.; Kabashin, A.V. Ultra-narrow surface lattice resonances in plasmonic metamaterial arrays for biosensing applications. Biosensors and Bioelectronics 2018, 104, 102-112.

(6) Riboh, J. C.; Haes, A. J.; McFarland, A. D.; Yonzon, C. R.; Van Duyne, R. P. A nanoscale optical biosensor: real-time immunoassay in physiological buffer enabled by improved nanoparticle adhesion. J. Phys. Chem. B 2003, 107, 1772-1780.

(7) Jung, L. S.; Campbell, C. T.; Chinowsky, T. M.; Mar, M. N.; Yee, S.S. Quantitative interpretation of the response of surface plasmon resonance sensors to adsorbed films. Langmuir 1998, $14,5636-5648$.

(8) Stuart, D.; Haes, A.; Yonzon, C.; Hicks, E.; Van Duyne, R. P. Biological applications of localized surface plasmonic phenomenae. IEE Proc.-Nanobiotechnol. 2005, 152, 13-32.

(9) Barbillon, G.; Bijeon, J.-L.; Bouillard, J.-S.; Plain, J.; Lamy de la Chapelle, M.; Adam, P.-M.; Royer, P. Detection in near-field domain of biomolecules adsorbed on a single metallic nanoparticle. J. Microsc.-Oxf. 2008, 229, 270-274. 
(10) Chen, S.; Svedendahl, M.; Käll, M.; Gunnarsson, L.; Dmitriev, A. Ultrahigh sensitivity made simple: nanoplasmonic label-free biosensing with an extremely low limit-of-detection for bacterial and cancer diagnostics. Nanotechnology 2009, 20, 434015.

(11) Larsson, E. M.; Langhammer, C.; Zoric, I.; Kasemo, B. Nanoplasmonic probes of catalytic reactions. Science 2009, 326, 1091-1094.

(12) Barbillon, G. Theoretical and experimental study of localized surface plasmon resonance biochemical nanosensors on metallic nanoparticles, Ph.D. thesis, Univ. of Troyes, France, 2007.

(13) Henderson, M.A. The interaction of water with solid surfaces: fundamental aspects revisited. Surface Science Reports 46, 2002, 1-308.

(14) Van Spronsen, M. A.; Weststrate, K.-J.; Den Dunnen, A.; Van Reijzen, M. E.; Hahn, C.; Juurlink, L. B. F. Hydrophilic Interaction Between Low-Coordinated Au and Water: $\mathrm{H}_{2} \mathrm{O} / \mathrm{Au}(310)$ Studied with TPD and XPS. The Journal of Physical Chemistry C 2016, 120 (16), 8693-8703.

(15) Gil, A.; Colchero, J.; Gómez-Herrero, J.; Baró, A.M. Macroscopic water deposits on polycrystalline gold measured by scanning force microscopy. Ultramicroscopy 2001, 86 (1-2), 19.

(16) Heras, J. M.; Albano, E. V. Adsorption of Water on Gold Films. A Work Function and Thermal Desorption Mass Spectrometry Study. Z. Phys. Chem. 1982, 129, 11- 20.

(17) Guo, L. Q.; Zhao, X. M.; Bai, Y.; Qiao, L. J. Water adsorption behavior on metal surfaces and its influence on surface potential studied by SPM. Applied Surface Science 2012, 258 (22), 90879091.

(18) Demirdjian, B.; Bedu, F.; Ranguis, A.; Ozerov, I.; Karapetyan, A.; Henry, C. R. Indirect Nanoplasmonic Sensing to Probe with a High Sensitivity the Interaction of Water Vapor with Soot Aerosols. The Journal of Physical Chemistry Letters 2015, 6 (20), 4148-4152.

(19) Chu, Y.; Schonbrun, E.; Yang, T.; Crozier, K. B. Experimental observation of narrow surface plasmon resonances in gold nanoparticle arrays. Appl. Phys. Lett. 2008, 93, 181108. 
(20) Jiang, H.; Li, T.; Ertorer, E.; Yang, J.; Sabarinathan, J.; Mittler, S. A biosensor based on periodic arrays of gold nanodisks under normal transmission. Sensors and Actuators A: Physical 2013, 189, 474-480.

(21) Zheng, Y. B.; Juluri, B. K.; Mao, X.; Walker, T. R.; Huang T. J. Systematic investigation of localized surface plasmon resonance of long-range ordered Au nanodisk arrays. Journal of Applied Physics 2008, 103, 014308.

(22) Ferry, D.; Picaud, S.; Hoang, P.N.M.; Girardet, C.; Giordano, L.; Demirdjian, B; Suzanne, J. Water monolayers on $\mathrm{MgO}(100)$ : structural investigations by LEED experiments, tensor LEED dynamical analysis and potential calculations. Surface Science 1998, 409 (1), 101-116. 


\section{SUPPORTING INFORMATION}

Supporting Information. Figures S1 to S2. Tables S1 to S2.

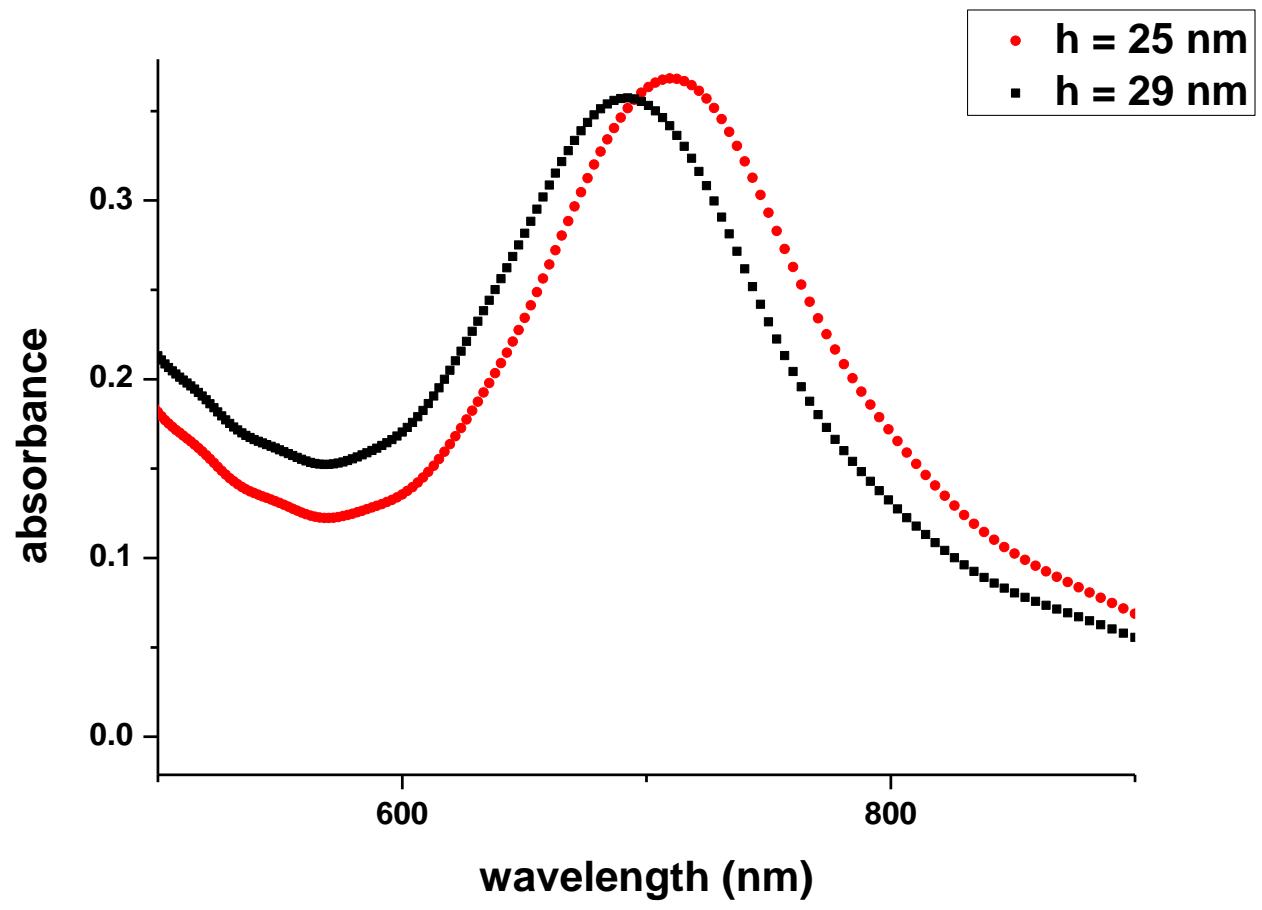

Figure S1: Theoretical LSPR responses (FDTD simulations) for gold nanodisks ( $p=300 \mathrm{~nm}, d=$ $150 \mathrm{~nm}$ ) deposited onto a borosilicate glass window vs the height $h$.

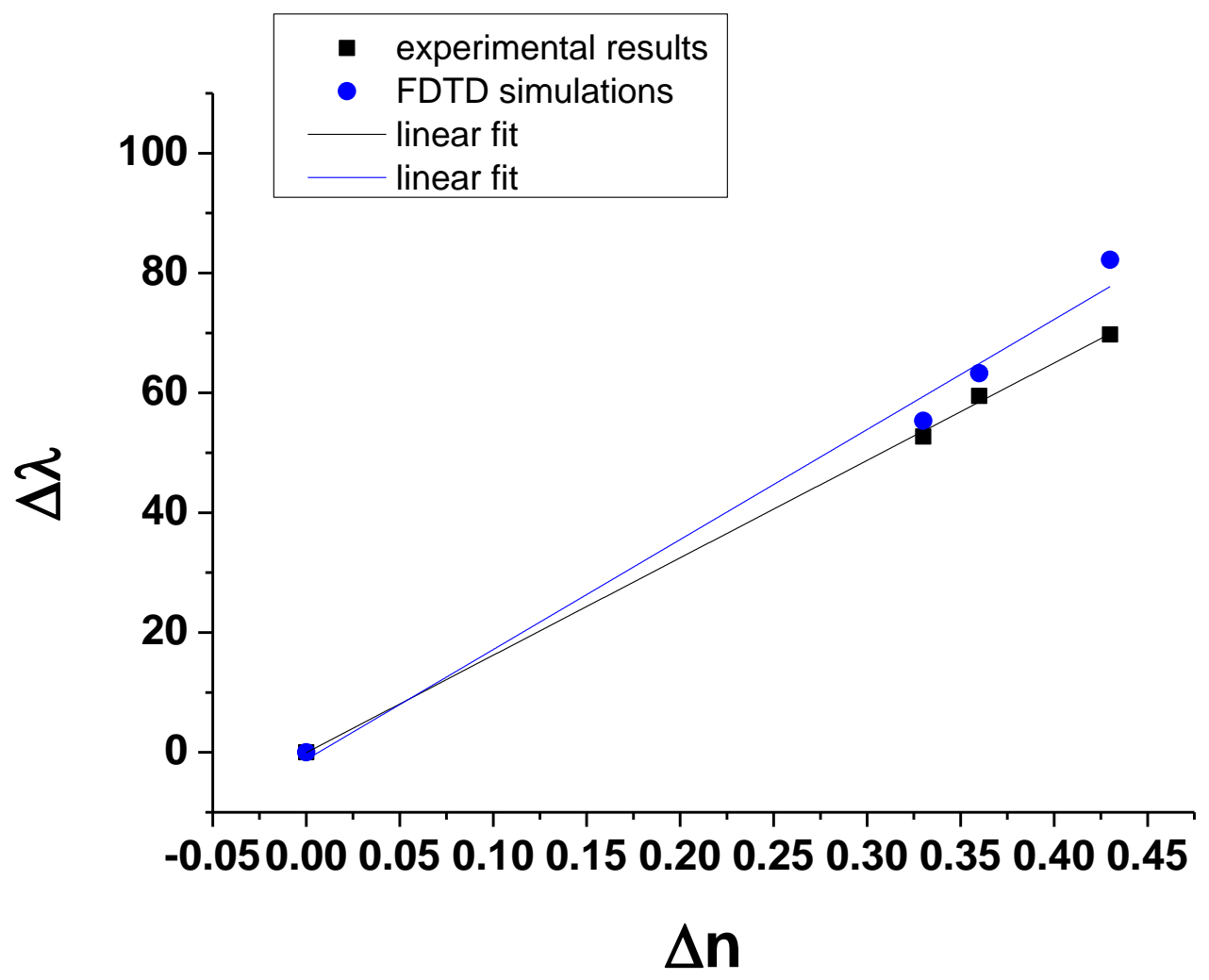


Figure S2: Experimental and theoretical (FDTD simulations) evolutions of the shift $\Delta \lambda$ of the LSPR response of gold nanodisks deposited on a borosilicate glass window vs the refractive index of the surrounding medium (air, water, ethanol, ethylene glycol).

Table S1: Simulated LSPR peak positions ( $\lambda$ ) and FWHM of the curves of the figure $2 a$

\begin{tabular}{|c|c|c|c|c|c|}
\hline$d$ & $p$ & $h$ & $d / h$ & $\lambda$ & $F W H M$ \\
\hline 100 & 250 & 25 & 4 & 653 & 112 \\
\hline 100 & 300 & 25 & 4 & 656 & 95 \\
\hline 100 & 350 & 25 & 4 & 664 & 91 \\
\hline
\end{tabular}

Table S2: Simulated LSPR peak positions ( $\lambda$ ) and FWHM of the curves of the figure $2 b$

\begin{tabular}{|c|c|c|c|c|c|}
\hline$d$ & $p$ & $h$ & $d / h$ & $\lambda$ & $F W H M$ \\
\hline 100 & 300 & 20 & 5 & 679 & 93 \\
\hline 100 & 300 & 25 & 4 & 656 & 95 \\
\hline 100 & 300 & 30 & 3 & 638 & 107 \\
\hline
\end{tabular}


TOC GRAPHICS

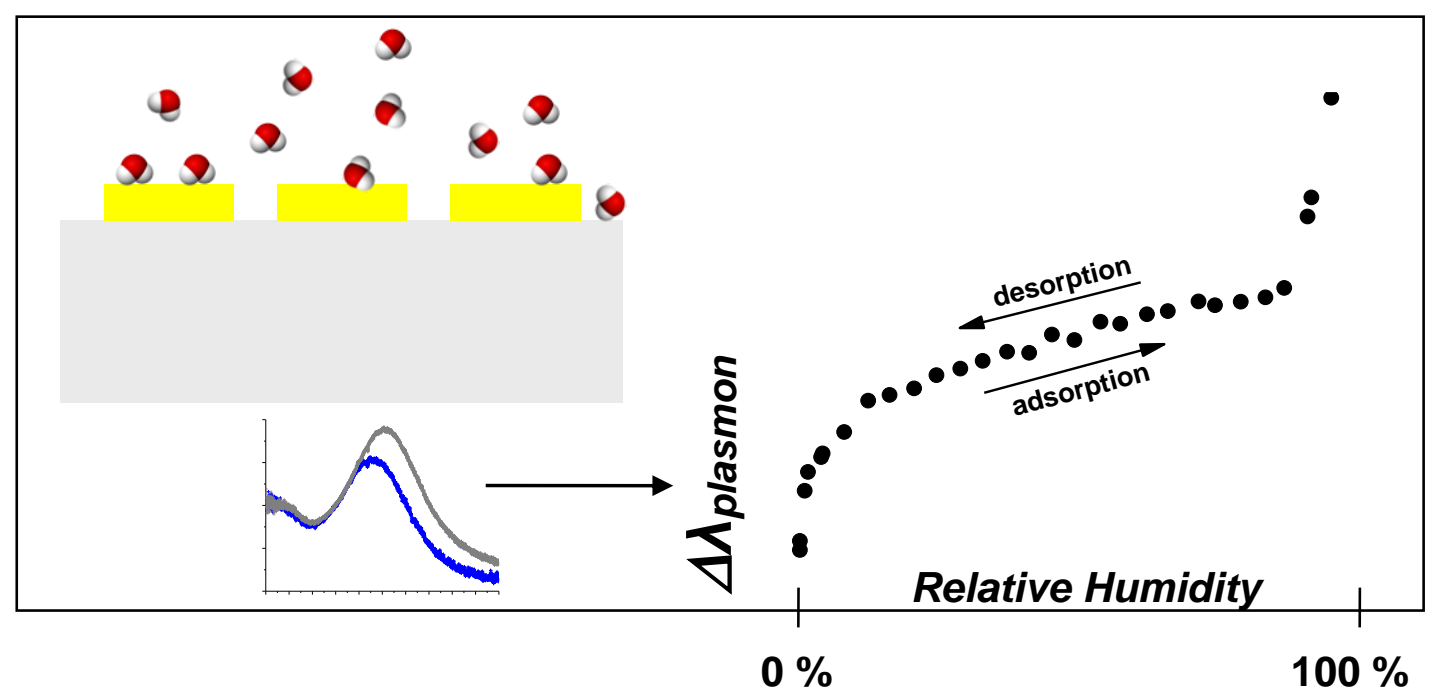

